\title{
PRESENCIA DE CONFLICTO ARMADO INTERNO Y SU EFECTO EN LA INVERSIÓN EXTRANJERA DIRECTA: TENDENCIA MUNDIAL Y PERSPECTIVAS PARA COLOMBIA (2001-2007)*
}

\author{
LUIS EDUARDO SANDOVAL** \& DEISSY MARTÍNEZ BARÓN**** \\ UNIVERSIDAD MILITAR NUEVA GRANADA
}

Recibido/Received/Recebido: 10/03/2009 - Aceptado/ Accepted/Aprovado: 15/01/2010

\begin{abstract}
Resumen
La identificación de una relación entre la presencia de conflicto armado interno (CAI) y la inversión extranjera directa (IED) mediante un modelo econométrico de regresión múltiple con datos panel es el objetivo central de este documento. Adicionalmente, se analiza el caso particular colombiano con el fin de determinar que tanta incidencia tiene la presencia de conflicto en el flujo de IED en su economía. Los principales hallazgos muestran una relación negativa entre los flujos de inversión extranjera directa en los países con conflicto armado interno y el gasto militar. Adicionalmente, se evidencia que el comportamiento de la IED en Colombia está determinado principalmente por las condiciones económicas y no por la situación de conflicto.
\end{abstract}

Palabras clave: Inversión Extranjera Directa, Conflicto Armado Interno, Datos panel.

\section{PRESENCE OF INTERNAL ARMED CONFLICT AND ITS INCIDENCE ON FOREIGN DIRECT INVESTMENT: GLOBAL TENDENCY AND PERSPECTIVES FOR COLOMBIA (2001 - 2007)}

\begin{abstract}
The objective of this paper is the identification of a link between internal armed conflict presence (IAC) and foreign direct investment (FDI) through an econometric model of multiple regressions with panel data is the main objective of this paper. The particular case of Colombia is analyzed with the purpose of determining if there is any incidence of armed conflict presence on FDI flows. Main results show a negative relation between foreign direct investment flows in countries with armed conflict and military expenditure. Additionally, the evidence shows that FDI behavior in Colombia is mainly determined by economic conditions and not conflict situation.

Keywords:Direct Foreign Investment, Internal Armed Conflict, Panel data.
\end{abstract}

Resultado del proyecto "Características Espaciales de Polarización y Concentración económica de los conflictos armados internos en el mundo durante 1980 - 2006".

Docente Investigador Programa de Economía. Facultad de Ciencias Económicas. Universidad Militar Nueva Granada. Correo Electrónico: luis.sandoval@unimnilitar.edu.co

*... Joven Investigadora Programa de Economía. Facultad de Ciencias Económicas. Universidad Militar Nueva Granada. Correo Electrónico: deissy.martinez@unimilitar.edu.co 


\title{
PRESENÇA DE CONFLITO ARMADO INTERNO E SEU IMPACTO SOBRE O INVESTIMENTO ESTRANGEIRO DIRETO: TENDÊNCIAS GLOBAIS E PERSPECTIVAS PARA A COLÔMBIA (2001-2007)
}

\begin{abstract}
Resumo
A identificação de uma relação entre presença de um conflito armado interno (CAI) e investimento estrangeiro direto (IED), utilizando um modelo econométrico de regressão múltipla com dados em painel é o objetivo central deste documento. Analisamos o caso colombiano para determinar o quanto impacto tem a presença de conflito no fluxo de IDE em sua economia. Os principais resultados mostraram uma relação negativa entre gasto militar e fluxo de IED em países com conflito armado interno. Além disso, o comportamento do IED na Colômbia é determinado pelas condições econômicas e não pelo conflito.
\end{abstract}

Palavras chave: Investimento estrangeiro direto, conflito armado interno, dados de painel

Sandoval, L. \& Martínez, D. (2010) Presencia de conflicto armado interno y su efecto en la inversión extranjera directa: tendencia mundial y perspectivas para colombia (2001-2007) En: Revista de la Facultad de Ciencias Económicas de la Universidad Militar Nueva Granada. rev.fac.cienc. econ, XVIII (1)

JEL: C13, C33, D74

\section{Introducción}

La presencia de Conflictos Armados Internos es una realidad que genera actualmente una serie de impactos políticos y económicos que se ven reflejados tanto a nivel nacional como internacional en las sociedades que los padecen. Dichos efectos en el ámbito económico se evidencian principalmente en variables de tipo macroeconómico como la Inversión Extranjera Directa (IED), la cual es objeto de estudio de este documento.

En este sentido, la IED explica en gran medida la fortaleza de las relaciones económicas de un país con los demás países, de igual manera, refleja el nivel de confianza que los inversionistas extranjeros tienen para invertir en un país determinado expresando estabilidad política y económica.

Por tanto, este documento pretende establecer, en primer lugar, la existencia de una relación entre la presencia de Conflicto Armado Interno y la IED, mediante una regresión múltiple con datos panel, que permitan relacionar diferentes variables a lo largo del tiempo a nivel mundial. En segundo lugar, se busca analizar si en Colombia el comportamiento de los flujos de IED están determinados por la situación de conflicto, por medio de una regresión lineal para el periodo 2000 - 2007. No obstante, dado que este documento es una primera aproximación es pertinente realizar un análisis juicioso con metodologías que permitan llegar a resultados que expliquen con mayor precisión la influencia del conflicto en la IED, como también en otras variables macroeconómicas.

El documento se desarrollará en cuatro partes esenciales, a saber: en primer lugar, la introducción, seguida del marco teórico en el cual se realiza una descripción general de la literatura revisada para su elaboración y de los términos relevantes para su comprensión. Una tercera parte consistirá en la evidencia empírica, la cual se encargará de especificar la metodología utilizada, las variables empleadas y la estimación junto con los resultados obtenidos y, finalmente, los comentarios finales. 


\section{Marco teórico}

Hasta el presente no se han realizado estudios profundos sobre la relación entre conflicto armado $e$ inversión extranjera directa, especialmente en países como Colombia que afronta una situación de conflicto, la cual a diario interfiere en el logro de un desarrollo y crecimiento económico que países similares, sin este tipo de problemática, si tienen. No obstante, se han realizado estudios principalmente en países desarrollados que analizan cómo la situación adversa de una sociedad afecta el desarrollo de ésta en el escenario económico.

Dado que el objeto de estudio es la inversión extranjera directa en países con conflicto armado interno, es necesario dejar estos dos conceptos claros: en primer lugar, la Inversión Extranjera Directa (IED), según el FMI, se da cuando un inversionista residente (inversionista directo), tiene como objetivo obtener una participación duradera en una empresa residente en otra economía o país (empresa de inversión directa) En Colombia, la IED se rige mediante el Artículo $3^{\circ}$ del Decreto 2080 de $2000^{1}$ mediante el cual se identifican cuatro modalidades: i) La adquisición de participaciones; ii) Acciones; iii) Cuotas sociales y; iv) Aportes representativos del capital de una empresa o bonos obligatoriamente convertib les en acciones.

En segundo lugar, la definición de Conflicto Armado Interno (CAI) tomada como referencia se encuentra en el Artículo $3^{\circ}$ común a los cuatro convenios de Ginebra y su Protocolo Adicional II de 1977, utilizada para la aplicación de la legislación internacional: "todos los conflictos armados que no estén cubiertos por el Artículo 1 del Protocolo adicional a los Convenios de Ginebra del 12 de agosto de $1949^{2}$ relativo a la protección de las víctimas de los conflictos armados internacionales (Protocolo I) y que se desarrollen en el territorio de una Alta Parte contratante entre sus fuerzas armadas y fuerzas armadas disidentes o grupos armados organizados que, bajo la dirección de un mando responsable, ejerzan sobre una parte de dicho territorio un control tal que les permita realizar operaciones militares sostenidas y concertadas". Ahora bien, una vez mencionadas las anteriores definiciones es pertinente revisar estudios relevantes para este análisis que conduzcan a un mejor entendimiento de los resultados.

\subsection{Inversión Extranjera Directa}

En resumen, la IED hace referencia a la participación económica de un inversionista en una empresa o compañía en otra economía, es decir, es aquella inversión que se realiza en un país diferente al de origen con el fin de obtener beneficios a mediano y largo plazo. Así pues, según Baracaldo (2004), los determinantes de la IED son cinco: las ventajas comparativas de cada localización, la geografía económica, los costos de comercio, la transferencia de tecnología y el tamaño de los mercados. A su vez, existen algunos factores que contribuyen también a la generación de movimientos de la IED, como las características únicas de cada país, las cuales tienen la posibilidad de provocar cambios positivos o negativos en este tipo de inversión.

En este sentido, Reuber et al. (1973) hace particular énfasis con respecto a que los principales determinantes de la IED en Estados Unidos son el mercado lucrativo, las políticas del gobierno receptor, infraestructura tecnológica, mano de obra calificada y la proximidad cultural, además de las variables tradicionales como el PIB, población, estabilidad política y económica, infraestructura y barreras débiles.

Por otro lado, Ramírez \& Flórez (2006) plantean una tipología diferente de las clases de inversión extranjera directa basada en las actividades que se realizan para internacionalizar empresas de una industria determinada, los tipos de IED que proponen son los siguientes: alianzas de riesgo compartido, compra de empresas existentes, montaje de empresas nuevas y por último, licencias y franquicias.

El estudio de la IED se ha analizado en distintas economías, especialmente en aquellas en vía de

Régimen General de Inversiones de Capital del Exterior en Colombia y de Capital Colombiano en el Exterior.

Es decir, conflictos de carácter internacional. [Disponible en Internet]: http://www.hchr.org.co/documentoseinformes/documentos/html/pactos/prot II adicional convenios ginebra.html 
desarrollo, por ejemplo Lalwani (2002) realiza un estudio en el cual considera la economía de catorce países, realiza un esfuerzo para evaluar el impacto de los flujos de inversión extranjera directa sobre los niveles de ahorro nacional e inversión. El autor comprueba con sus hallazgos que las economías asiáticas tienen un muy alto promedio en tasas de inversión y ahorro comparado con los países latinoamericanos. Adicionalmente, la influencia de la inversión extranjera directa en ahorro e inversión es positiva únicamente en las economías de Chile, Corea y Tailandia, mientras que en Argentina y Filipinas la influencia es negativa.

A renglón seguido, se realiza una descripción general del comportamiento de tres variables macroeconómicas de los países con presencia de conflicto en conjunto, por cada continente. Las variables macroeconómicas que se analizan son: la Inversión Extranjera Directa $^{3}$ (IED) (tomada en la estimación econométrica como variable dependiente), el crecimiento del Producto Interno Bruto 4 y el ingreso per cápita ${ }^{5}$, variables que proporcionan un panorama general de la economía de los países que presentan conflicto.

\subsection{Relación Conflicto Armado Interno y Economía}

A pesar de la presencia de conflicto armado interno en Colombia, su análisis y estudio a nivel académico se ha desarrollado intensamente tan solo hasta la última década. No obstante, el tema ha sido estudiado en otros países, principalmente en la academia norteamericana y europea. Lo mismo, el análisis de los conflictos armados que se han presentado a lo largo de la historia es realizado generalmente en el plano de lo político. Sin embargo, en los últimos tiempos se ha integrado el concepto económico al análisis, lo cual ha permitido avanzar profundamente en la explicación y desarrollo de este tipo de conflictos considerando variables económicas de los países afectados como el producto interno bruto, inversión extranjera directa, crecimiento, entre otros.

En este sentido, se han planteado teorías que buscan encontrar un patrón de comportamiento compuesto de la unión de diferentes factores que incidan en la presencia o no de un conflicto armado interno, es así como un autor colombiano, Restrepo (2001), plantea que los conflictos radican en países cuyos ingresos son bajos, es decir, aquellos países que se clasifican como pobres a nivel internacional. $\mathrm{El}$ autor acierta por lo menos en el caso colombiano, considerando la importancia de analizar otros aspectos que componen la realidad del conflicto en Colombia, lo cual se realizará más adelante.

No obstante, en naciones donde la democracia es ejercida mediante voto popular y representación parlamentaria, como Somalia, Chad, República Centroafricana y Colombia, existen grupos que justifican su militarización en la inconformidad por la posición que ocupan dentro de la sociedad.

Como se mencionó anteriormente, el plano político dejó de ser la plataforma única sobre la cual el tema de los conflictos armados internos es analizado. De esta manera, algunos estudios económicos sobre conflictos internos muestran el impacto de éstos en el sector externo de las economías.

Tomando como variables principales el comercio internacional y las guerras civiles ${ }^{6}$, dichos estudios concluyen que existe un impacto en diferentes niveles sobre el comercio bilateral debido a las guerras civiles, impacto que es de carácter negativo, donde el nivel de comercio internacional es menor si el país afectado posee alianzas. Igual, una caída en el

3 Es la inversión cuyo objeto sea adquirir una participación permanente y efectiva en la dirección de una empresa en una economía que no sea la del inversionista, según el FMI.

4 El crecimiento del Producto Interno Bruto se considera como la variación del crecimiento del Producto Interno Bruto (PIB) de un año a otro, siendo el PIB el valor total de los bienes y servicios finales producidos en el territorio de un país, por residentes nacionales y extranjeros, en un periodo determinado. Fuente: Apartado estadístico del Banco Mundial

5 Valor total de la producción corriente de bienes y servicios finales dentro del territorio nacional durante un cierto período de tiempo, dividido por el valor de la población total.

6 Una guerra civil es un conflicto que ocurre generalmente dentro de las fronteras de un país, en ocasiones con intervención extranjera. 
comercio bilateral se ve asociado a la interacción de las variables independientes relacionadas con la guerra civil (Bayer \& Rupert, 2004).

En un estudio realizado por Nigh \& Schollhammer (1987), los eventos políticos, la situación de conflicto y las asimetrías sociales son variables utilizadas para determinar el nivel de impacto de la situación de conflicto en la inversión extranjera directa. Estos autores tienen especial importancia en este documento debido a que son los primeros que aproximan una relación entre el conflicto y la IED. En su estudio, observan el efecto y la capacidad de respuesta en Japón frente a países desarrollados y subdesarrollados. Dicho análisis, concluye que los inversionistas responden con mayor fuerza al aumento del conflicto que a la disminución del mismo, a pesar de que una disminución del conflicto implica generalmente mayor desarrollo y crecimiento económico.

\subsection{Caso particular de la IED y el CAI}

El análisis de Nigh \& Schollhammer (1987) deja entrever la importancia del papel de la IED en el crecimiento y desarrollo económico de un país, así como los efectos que tienen en la economía, los cuales giran entorno a la alteración en los beneficios y la posibilidad de afectar la economía aumentando el riesgo, es decir, al desviar la estrategia de cobertura perfecta en la inversión el riesgo tiende a aumentar. Ahora, una de las soluciones planteadas es el mayor aprovechamiento de las oportunidades de inversión y de producción en un país en donde las condiciones sean atractivas (Calderon \& Rossell, 1985). De este modo, la inversión extranjera directa puede ser relacionada con el crecimiento económico. Baracaldo et al. (2005) sostiene en un estudio que la IED tiene efectos positivos sobre el crecimiento económico, independientemente del grado de desarrollo de las economías, su ubicación geográfica y demás consideraciones propias a cada economía.

Para el caso colombiano, Lombard (1978) analizó la inversión extranjera y el proceso de toma de decisiones en países de menor desarrollo con mercados aún sin explotar ubicados en Latinoamérica y particularmente en Colombia. El autor concluye que el caso colombiano refleja una gran diferencia entre las regulaciones en la proyección hacia los mercados y las prácticas administrativas, que puede ser explicado por la subjetividad en la toma de decisiones, así como la concentración de poder en un bajo porcentaje de la población.

El comportamiento de la IED en Colombia se debe a innumerables factores. Sin embargo, dicho comportamiento no ha sido analizado desde la perspectiva del conflicto, conflicto que de todas formas se debe a diversos factores, entre ellos, la sostenibilidad financiera de los grupos armados al margen de la ley en el narcotráfico y prácticas que violan los derechos humanos como el secuestro (Pizarro, 2004).

Más aún, en naciones donde la democracia ha sido instaurada y es ejercida mediante voto popular y representación parlamentaria, como es el caso de Somalia, Chad, República Centroafricana y Colombia, existen grupos que justifican su militarización en la inconformidad por la posición que ocupan dentro de la sociedad. Los conflictos existentes durante el periodo analizado serán relacionados a continuación, los cuales han sido clasificados con base en la literatura y bases de datos internacionales ${ }^{7}$ de conflictos.

En este sentido, Valencia (2006) corrobora lo dicho por Pizarro (2004) considerando que los actores del conflicto adoptaron después de los 90s una lógica económica que viene enfatizando en la consecución de medios de financiación a través de la protección de actividades relacionadas con los cultivos ilícitos, percibiendo por ello retribuciones económicas de narcotraficantes, convirtiendo este tipo de recursos en el combustible del conflicto colombiano.

\section{Evidencia empírica: flujos de IED en países con $\mathrm{CAI}^{8}$}

Una vez realizada la revisión de los principales estudios que relacionan la economía con situaciones de

\footnotetext{
7 Las fuentes utilizadas en la realización del documento son: Banco Mundial, Fondo Monetario Internacional, Journal of Peace Research, Departamento Nacional de Estadística, Departamento de Planeación Nacional, Proexport.

8 Los datos relacionados en este apartado son extraídos de las bases de datos del Banco Mundial.
} 
conflicto y en particular, la inversión extranjera directa, es pertinente analizar no sólo los flujos de inversión extranjera sino también el producto interno bruto per cápita, la cual es una variable macroeconómica que permite observar en términos generales el estado de la economía en la sociedad de un país.

\subsection{Flujos de IED y PIB per cápita de los países con CAI}

En este apartado se realizará el análisis descriptivo de los flujos de IED en los países con presencia de conflicto armado interno, agrupados por los continentes a los cuales pertenecen. En el análisis se incluye también el comportamiento del producto interno per cápita con el fin, de observar el nivel de ingreso que hay en los países que tienen este tipo de conflicto.

\subsubsection{Asia}

Es el continente más grande del mundo, tiene la mayor concentración de población del planeta y una economía con diferentes características en cada una de las regiones que lo componen. Existen países desarrollados y poderosos, y otros pobres con escasos recursos. Los países de Asia son, en su mayoría, atractivos para los inversionistas extranjeros. En el año 2004 India y China estuvieron dentro el ranking de los 10 países de Asia-pacifico con mayor flujo de IED, según la agencia de rating Standard \& Poor's. Este hecho se debió al marco financiero global, los aumentos de los beneficios empresariales, la creciente confianza en las políticas económicas que están aplicando mercados emergentes como China, así como la tendencia de liberalización de los movimientos de capital y las políticas de atracción de inversión extranjera aplicadas por economías como India y China (Martin, 2005).

La inversión extranjera directa en China supera por más de US\$30.000 millones de dólares el resto de países asiáticos con presencia de Conflicto Armado Interno, en donde, ni siquiera India, uno de los paí- ses que está dentro de los 10 países con mayor IED, está al nivel de China.

La mayoría de los países de Asia se encuentran en nivel de ingreso medio, según la clasificación de Restrepo (2001) ${ }^{9}$. En la medida que el ingreso per cápita de países como China e India está por debajo del promedio a pesar de ser los países con un crecimiento ascendente y con presencia de conflicto en el periodo analizado.

Igualmente, el crecimiento de países como Iraq es atípico y sobrepasa el promedio del resto de países asiáticos, situación que se debe a los índices de inflación que se evidenciaron particularmente en Iraq después de la caída del gobierno de Saddam Hussein, periodo en el cual la escasez en los alimentos generó un incremento sustancial en los precios y el nivel de vida en general. En cuanto a Israel, a pesar de que su crecimiento del PIB no avanza con tasas importantes, es el único país con ingreso per cápita alto en donde hay presencia de Conflicto Armado Interno.

\subsection{2. África}

El continente africano es uno de los más aislados del comercio mundial. Al mismo tiempo afronta diversos frentes destructivos como crisis humanitarias, económicas y conflictos tanto internos como interestatales. Estos factores han impedido que la región consiga crecimiento, desarrollo económico e integración económica, además de política con el resto de la comunidad internacional.

Por otro lado, la atracción de los recursos naturales de África explica la distribución desigual de las corrientes de IED en el continente. Los 24 países africanos clasificados por el Banco Mundial como dependientes del petróleo y los minerales, han atraído un promedio de casi las tres cuartas partes de las corrientes anuales de IED en los últimos dos decenios $^{10}$.

9 Restrepo (2001), hace una clasificación basada en los ingresos de todos los países, en quintiles, los cuales son ingrsesos muy bajos, bajos, medios, medios altos y altos.

10 UNCTAD, (Conferencia de las Naciones Unidas sobre comercio y desarrollo). "La inversión extranjera directa en África". En: Publicaciónes.[Disponible en Internet] http://www.unctad.org/templates/Page.asp?intItemID=3524\&lang=3. 
Según la UNCTAD (2005), la especial combinación de características geográficas, históricas y estructurales de África han hecho que la IED se concentre habitualmente en enclaves de producción primaria orientada a la exportación con escasos vínculos con el resto de la economía.

La situación de los países africanos en su gran mayoría se basa en una economía de subsistencia. Sudáfrica es la excepción pues tiene un importante papel dentro del continente como a nivel mundial y posee una economía de ingresos medios basada en la extracción de minerales como diamantes, oro, y platino. Los demás países de África conservan condiciones propias de subdesarrollo, especialmente en la región subsahariana, en donde carecen de infraestructuras de transportes, medios técnicos de producción, industria y sistemas financieros.

\subsubsection{América}

El continente americano gira entorno a una de las economías más grandes y fuertes del mundo (caso de EE.UU.) y una de las economías más pobres (Haití). Los únicos países que permanecen con conflicto interno en este continente son Colombia y Haití. En este apartado se hará referencia tan solo al caso haitiano, toda vez que para Colombia se dedicará una sección.

La economía haitiana se mantiene en gran parte por la agricultura de subsistencia y se encuentra en la posición 150 de 177 países en el Índice de Desarrollo Humano de la ONU ${ }^{11}$, mientras que la economía colombiana, a pesar del conflicto que presenta, mantiene una economía estable con alta participación de inversión extranjera directa en el $\mathrm{PIB}$, una balanza comercial estable y tendiente a la diversificación.

La inversión extranjera directa en Haití ha venido en aumento debido a la paulatina y lenta liberación de las restricciones para los inversionistas extranjeros, considerando que el gobierno debe autorizar determinadas transacciones de inversión para que puedan ser realizadas. Esto se evidencia en el ranking de libertad económica donde el país ocupa el puesto 138 y en cuanto a libertad de inversión tan solo registra un 30\% (Index of Economic Freedom, 2008). La economía haitiana es la menos desarrollada del hemisferio occidental, aproximadamente un $70 \%$ de la población vive en la pobreza.

\subsubsection{Caso Colombiano}

\section{Características generales del conflicto}

Colombia tiene un conflicto de varias décadas, cuyos principales actores se ubican en dos extremos: por un lado están FARC, ELN y los paramilitares, y por otro, el Estado, el cual tras cada gobierno ha intentado recuperar la soberanía total del territorio mediante presencia militar. El conflicto colombiano
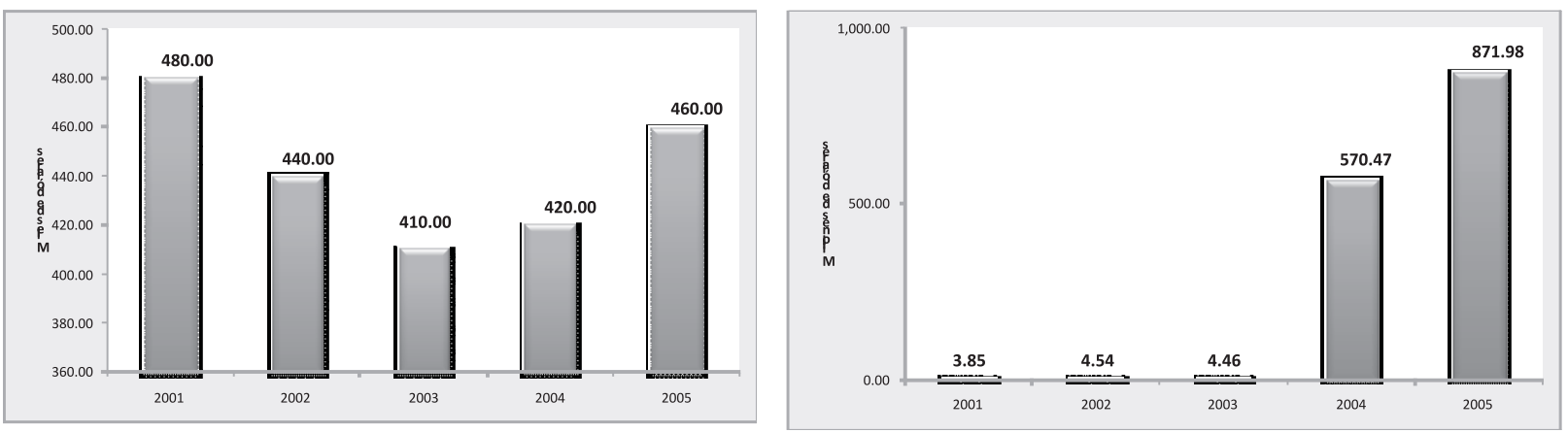

Ilustración 1. Flujos de IED e Ingreso per Cápita de Haití12

11 Escala de IDH (Índice de Desarrollo Humano para 177 países) Organización de Naciones Unidas ONU.

12 Cálculos realizados por el autor a partir de datos estadísticos del Banco Mundial y FMI. 
tiene tres componentes esenciales de análisis que aportan a la configuración de los actores y su comportamiento $\mathrm{y}$, además permite entender, la situación del conflicto en los últimos años.

En primer lugar, los grupos al margen de la ley en Colombia no tienen una potencia detrás de su accionar; en segundo lugar, los métodos utilizados por los actores no estatales son crueles y criminales, y por último, la financiación de estos grupos recae principalmente en el narcotráfico, en sus distintas etapas (Borrero, 2006). Estos componentes de análisis permiten visualizar globalmente la complejidad del conflicto en Colombia, considerando marco internacional, interno y el económico de los grupos insurgentes colombianos.

\section{La IED y la economía Colombiana}

La economía colombiana se ha desarrollado en el sector de servicios con gran eficiencia y rapidez, convirtiéndola en una de las economías con mayor atracción de capital extranjero, lo que se ve en el aumento del producto, por encima de su nivel de pleno empleo macroeconómico evidenciado con tasas de crecimiento del $6 \%{ }^{13}$ que se sustenta en una fuerte expansión de importantes elementos de la demanda agregada, como es el caso de la inversión privada, y de un buen desempeño de sectores estratégicos en la producción nacional (López M. et al. 2006).

De igual forma, Colombia ha tenido un crecimiento económico estable, aunque en el año 2000 y 2001 el crecimiento fue rezagado, la rápida recuperación ha sido evidente en los últimos años (ver ilustración 2). Uno de los componentes económicos que ha influido en este comportamiento de la economía es la inversión extranjera directa, la cual ha tenido una tendencia al alza debido al retorno significativo de la confianza que el país genera hacia los inversionistas en el resto del mundo.

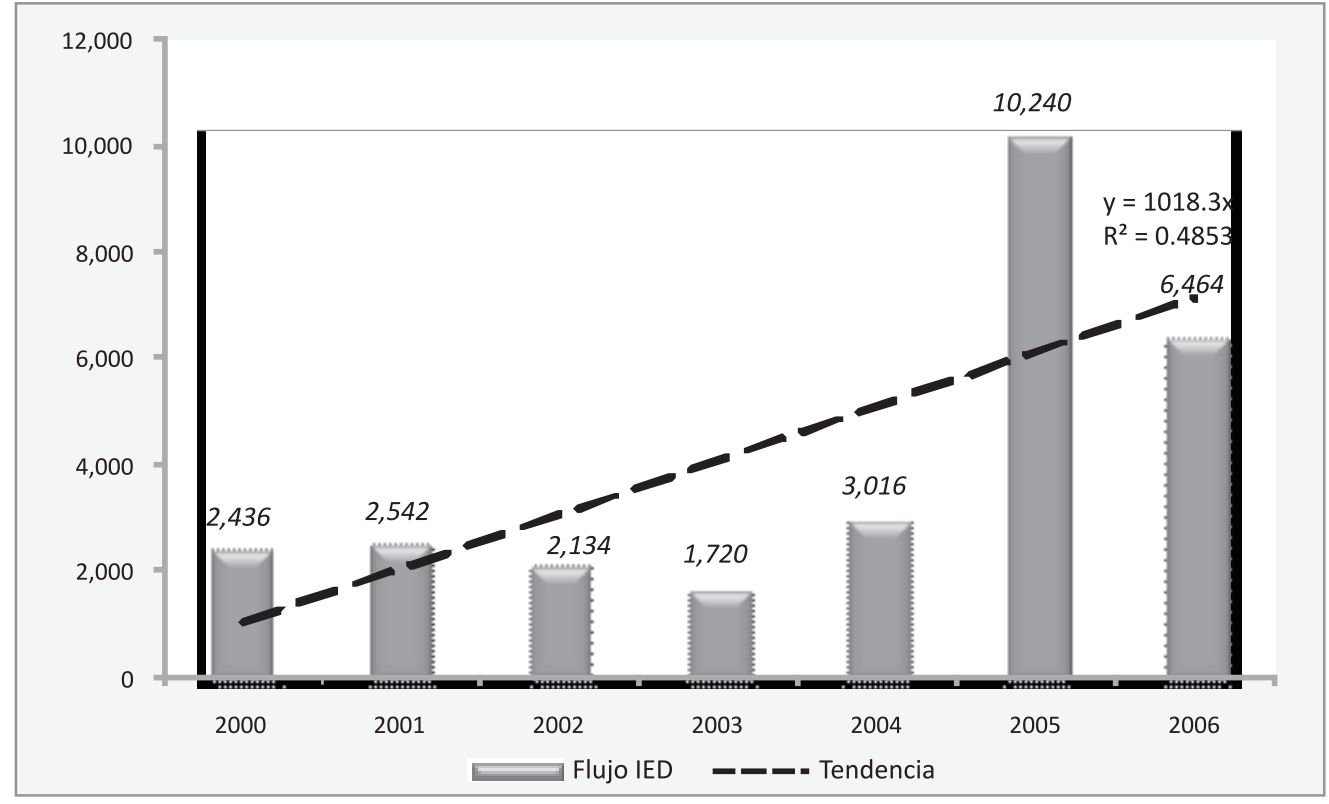

llustración 2. Flujos de IED en Colombia ${ }^{14}$

Estadísticas del Banco de la República.

Cálculos realizados por el autor con datos del Banco de la República. 
El comportamiento de la IED en Colombia ha tenido un comportamiento creciente con variaciones leves en el periodo 2000-2004 (ver ilustración 3). Sin embargo, el siguiente año tuvo un ascenso extraordinario debido a la compra de Bavaria por SabMiller, teniendo en cuenta que el aporte de esta transacción fue de US $\$ 4.715$ millones de los US $\$ 10.240^{15}$ registrados para ese año. Según BusinessWeek, Colombia tiene como fortaleza la economía y su dinámica para atraer inversión extranjera, así como una política económica que ha sido seria y responsable a lo largo de la historia, evidenciada en hechos como el cumplimiento ininterrumpido de los pagos a la deuda externa del país.

El crecimiento de la economía colombiana ha sido importante desde el 2001-2002, sobre todo en los últimos años, soportándose en los sectores de la construcción, manufactura y comercio. La situación económica de Colombia está mejorando, pero no lo suficiente para desmotivar a las guerrillas a desvincularse de la guerra, cuya sostenibilidad está en las drogas, las cuales se convirtieron en un negocio de alta rentabilidad, lo suficiente para sostener una organización armada (Pizarro, 2004) y hacer daño a la estructura económica nacional. Habiendo tenido una descripción general de las principales características económicas y la situación de Conflicto
Armado Interno en Colombia, en la siguiente sección se muestra la evidencia empírica realizada para cumplir con el propósito del documento.

\section{Descripción del modelo y resultados}

El objetivo central del documento es identificar si existe una relación entre el conflicto y la IED, demostrando que la presencia de Conflicto Armado Interno incide negativamente en la IED, mediante la inclusión de países sin conflicto armado interno. Adicionalmente el documento pretende hacer un análisis de la IED en el contexto colombiano con el fin de demostrar que la IED es creciente a pesar de la situación de conflicto.

Por tanto, el modelo econométrico más apropiado para satisfacer el objetivo del documento es una regresión múltiple con datos panel, modelo que permite hallar relaciones más precisas con datos no sólo de corte transversal sino también series de tiempo, a continuación se explica el modelo planteado y la metodología en detalle.

\subsection{Planteamiento del modelo y metodología}

La metodología utilizada busca hacer un primer acercamiento al análisis, sujeta a modificaciones que
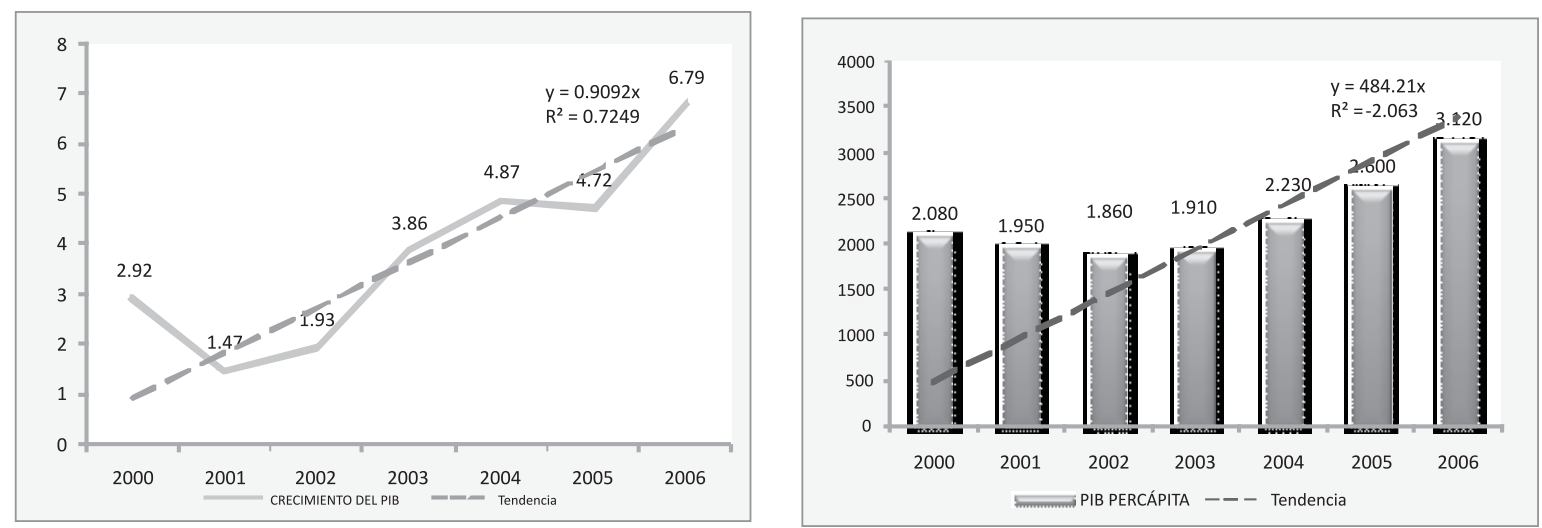

Ilustración 3. Crecimiento del PIB e Ingreso Per cápita de Colombia ${ }^{16}$

\footnotetext{
15 Según datos de Proexport.

16 Cálculos realizados por el autor con datos del Banco de la República.
} 
garanticen un análisis más profundo y que muestre resultados más determinantes en versiones posteriores del documento. En este sentido, con el fin de comprobar la primera hipótesis, se utilizará un modelo de regresión múltiple con datos panel para el periodo 2001-2007. Los datos en panel utilizan dos dimensiones: el tiempo y el espacio, por esta razón, agrupan y combinan datos tanto transversales como datos de series de tiempo. Las variables empleadas en la estimación son variables de conflicto (intensidad, naturaleza y ubicación) y socioeconómicas (como la tasa de desempleo, de crecimiento, de alfabetismo, inflación, entre otras), de manera que, permitan establecer la relación con la inversión extranjera directa. El modelo planteado para la estimación es el siguiente:

$\operatorname{lnied}_{i t}=\alpha_{i t}-$ gmil $_{i t}+$ intb $_{\text {it }}-$ intm $_{\mathrm{it}}$

El Test de Hausman es una prueba que permite determinar el método apropiado para estimar la regresión, el método de efectos fijos ${ }^{17}$ o mediante el método de efectos aleatorios ${ }^{18}$. El análisis se inicia estimando el modelo subyacente utilizando mínimos cuadrados ordinarios luego de combinar los datos de corte transversal con datos de series de tiempo. Posteriormente, se identifican variables que deben ser omitidas porque no contribuyen a la significancia de la estimación, sin ignorar que tales variables pueden conducir a cambios en los interceptos del corte trasversal y las series de tiempo.

Existen diversos modelos para datos panel cuya estructura esencial en la mayoría de ellos es un modelo de efectos:

$Y_{i t}=\alpha_{i}+y_{i}+\beta^{\prime} X_{i t}+\varepsilon_{i t}$

Esta función representa tanto el método de efectos fijos como el método de efectos variables, donde eit es el error que debe cumplir lo siguiente: $\varepsilon^{\prime}\left[\varepsilon_{i t}\right]=0 ; \operatorname{Var}\left[\varepsilon_{i t}\right]$

Una vez que las pruebas econométricas sean realizadas y el modelo sea estimado, se espera que las estimaciones muestren resultados que conlleven a la comprobación de las hipótesis planteadas en el documento.

\subsection{Variables empleadas}

Las variables utilizadas para realizar la regresión con datos panel fueron clasificadas en variables de conflicto, económicas y sociales. La determinación de estas variables se hizo con base en la revisión de literatura realizada y análisis de autores como Gleditsch et al. (2002, 2005), Lacina (2004), Murdoch \& Sandler (2002), Pickering \& Kisangani (2006), Reynal-Querol (2002), Sambanis (2001) solo por mencionar algunos, quienes se inquietan por determinar las relaciones, influencias y conexiones de las situaciones sociales de conflicto y el comportamiento económico. Las variables de conflicto son cuatro. La primera es la variable que determina la presencia o no de conflicto en los países, es una variable dicotómica en donde 0 corresponde a la no presencia de conflicto y 1 corresponde a la presencia de conflicto en determinado país, variable que esta designada como (conf) en la tabla de resultados.

La segunda y tercera variables aluden a la intensidad del conflicto. Los estudios revisados como determinan que existen tres categorías de intensidad (alta, media y baja), por lo que se tomó una primera variable dicotómica en donde 0 y 1 corresponden a la no presencia de conflicto $y$, la presencia de conflicto de intensidad baja, respectivamente fue denominada (intb). De igual forma se construyó la segunda variable de intensidad, en la cual 0 corresponde a la no presencia de conflicto y 1 hará referencia a la presencia de conflicto de intensidad media y alta, (intm).

17 El método de Efectos Fijos permite agregar variables indicadoras que permitan que el término del intercepto varíe a lo largo del tiempo y a lo largo de las unidades del corte trasversal (Pindyck, 1998).

18 El Método de Efectos Aleatorios toma un modelo de corte trasversal y series de tiempo combinados, en el que los términos del error pueden correlacionarse a lo largo del tiempo y de las unidades individuales. Este modelo puede estimarse con una regresión de mínimos cuadrado generalizados, (Pindyck, 1998). 
La cuarta variable corresponderá al porcentaje del Producto Interno Bruto destinado al gasto militar ${ }^{19}$ en los países con y sin conflicto. Esta variable de conflicto es importante puesto que muestra la relación entre capacidad militar y la necesidad de destinar recursos para las fuerzas armadas de cada país, en muchos casos, dejando de invertir en otros rubros importantes para la sociedad y el desarrollo de la misma.

Las variables económicas utilizadas en la estimación son seis, entre las que se encuentran la inflación (inf), el crecimiento del Producto Interno Bruto (PIB) (cpib), exportaciones (xpib) e importaciones (mpib) como porcentaje del PIB, índice de precios al consumidor (ipc) y deuda externa (dext). Adicionalmente, se utilizaron dos variables sociales dentro de la estimación, que son la tasa de alfabetismo (talf) y la tasa de crecimiento de la población (tcpob).

La segunda hipótesis será comprobada mediante una regresión lineal, para el periodo 2000-2006 trimestral. El periodo fue extendido con el fin de buscar robustez en los datos, utilizando variables como la tasa de interés de colocación, la tasa de interés activa, tasa de interés - DTF, Formación Bruta de Capital (FBK), población, crecimiento del PIB, empleo, desempleo, balanza comercial y coeficiente de Gini.

El modelo planteado para la comprobación de la segunda hipótesis se establece a continuación, donde la variable dependiente la IED tiene relación con la Tasa de interés (DTF), la Formación Bruta de Capital (FBK), la balanza comercial, la tasa de desempleo y empleo, y el producto interno bruto.

$\ln$ ied $_{i t}=\alpha_{i t}+t i d t f_{i t}+f b k_{i t}+\operatorname{lnbcom}_{i t}-$ tdes $_{i t}$ + temp $_{i t}+\ln$ ib $_{i t}$
Al realizar la estimación se espera que los resultados comprueben la existencia de una relación positiva entre la IED y todas las variables incluidas en el modelo, a excepción de la Tasa de Desempleo, ya que al existir desempleo la actividad y estabilidad económica no genera confianza para la inversión.

La estimación de la regresión lineal para el caso colombiano se realizó utilizando variables tanto económicas como de carácter social. Las variables económicas empleadas son: inversión extranjera directa (variable dependiente) (lnied), tasa de interés activa (tiact), las remesas (rem), la tasa de interés (tidtf), producto interno bruto (lnpib), exportaciones (lnexp) e importaciones (lnimp) para el periodo 2000-200720.

\subsection{Resultados}

Se realizaron dos estimaciones con el fin de observar las posibles variaciones en los resultados de una estimación a otra. La primera estimación se realizó con el método de efectos fijos (fixed effects) y la segunda se realizó entre efectos (between effects). En los resultados se encontraron variaciones en algunas de las variables, en la significancia y en algunos signos que cambiaron de una estimación a otra. Los resultados muestran que el porcentaje del PIB destinado al gasto militar es significativo y tiene una relación positiva con la inversión extranjera directa (Tabla 1.) debido a que el comportamiento de la IED es determinado en gran medida por la confianza y estabilidad que exista en un país. Al haber gasto militar en un país con presencia de conflicto se genera una sensación de estabilidad y confianza hacia los inversionistas extranjeros, ya que esto representa la preocupación y la intención del gobierno por proveer seguridad y generar un buen ambiente económico.

19 Esta variable incluye todos los gastos de capital y corrientes en las fuerzas militares, incluyendo fuerzas de mantenimiento de la paz, ministerios de defensa y otras agencias gubernamentales relacionadas con proyectos de defensa. Datos de gasto militar tomados del Banco Mundial a partir de la definición establecida por la OTAN.

20 A las variables IED, PIB, Exportaciones, Importaciones y Balanza Comercial se les calculó el logaritmo natural con el fin de normalizar los datos y así evitar sesgos en la estimación. 
Tabla 1. Resultados - Variable dependiente LnIED²1

Variable dependiente: Logaritmo Natural IED

\begin{tabular}{|c|c|c|}
\hline \multirow[b]{2}{*}{ Variable } & \multicolumn{2}{|c|}{ Estimaciones } \\
\hline & $1 *$ & $2^{\star \star}$ \\
\hline \multirow[t]{2}{*}{ conf } & -285000000 & $-2,87 E+08$ \\
\hline & $(0,411)$ & $(0,391)$ \\
\hline \multirow[t]{2}{*}{ gmil } & $-0,8215579$ & 0,542637 \\
\hline & $(0,000)$ & $(0,244)$ \\
\hline \multirow[t]{2}{*}{$\inf$} & 1690084 & 2577787 \\
\hline & $(0,250)$ & $(0,690)$ \\
\hline \multirow[t]{2}{*}{ dext } & 0,0197651 & 0,0165881 \\
\hline & $(0,018)$ & $(0,100)$ \\
\hline \multirow[t]{2}{*}{ pib } & 0,0054112 & $-0,0064401$ \\
\hline & $(0,000)$ & $(0,000)$ \\
\hline \multirow[t]{2}{*}{$\exp$} & 0,0096063 & $-0,1981062$ \\
\hline & $(0,000)$ & $(0,000)$ \\
\hline \multirow[t]{2}{*}{ imp } & 0,1425476 & 0,3034084 \\
\hline & $(0,000)$ & $(0,000)$ \\
\hline \multirow[t]{2}{*}{ pobla } & $-3,374210$ & $-2,269436$ \\
\hline & $(-0,128)$ & $(-0,255)$ \\
\hline \multicolumn{3}{|c|}{ P-valor en paréntesis } \\
\hline \multicolumn{3}{|c|}{${ }^{\star}$ Fixed effects **Between effects } \\
\hline \multicolumn{3}{|c|}{ Nivel de significancia de las estimaciones 5\% } \\
\hline
\end{tabular}

La deuda externa tiene una relación positiva y significativa con la IED, teniendo en cuenta que puede explicar la capacidad de endeudamiento que tiene un país lo hace atractivo a los inversionistas. De igual forma el PIB, las exportaciones e importaciones tienen relación positiva y significativa con la variable dependiente, esto explicado por la mayor atracción que tiene hacia un inversionista un país cuyos niveles de crecimiento son determinantes en la atracción de inversión extranjera directa.

En el caso colombiano se realizaron dos estimaciones con el mismo propósito establecido para el caso a nivel mundial. La primera estimación se realizó mediante una regresión lineal (linear regression) convencional y la segunda, por medio de una regresión robusta (robust regression). Los resultados obtenidos evidencian la relación positiva entre la tasa de interés activa. De igual modo, las variables relacionadas en el comercio con el resto del mundo, las exportaciones e importaciones resultaron tener una relación significativa con respecto a la IED en la regresión robusta. La relación con las exportaciones es positiva en relación a la IED, mientras que las importaciones se relacionan positivamente con la variable dependiente.

21 Datos fuente. Datos tomados de Banco Mundial y FMI, cálculos de los autores 
Tabla 2. Resultados - Variable dependiente LnIED 22

Variable dependiente: Logaritmo Natural IED

\begin{tabular}{|c|c|c|}
\hline \multirow[b]{2}{*}{ Variable } & \multicolumn{2}{|c|}{ Estimaciones } \\
\hline & $1^{*}$ & $2^{* \star}$ \\
\hline \multirow[t]{2}{*}{ tia } & $-0,0029765$ & 11,925 \\
\hline & $(0,073)$ & $(0,701)$ \\
\hline \multirow[t]{2}{*}{ rem } & 0,0955178 & 0,0074815 \\
\hline & $(0,417)$ & $(0,984)$ \\
\hline \multirow[t]{2}{*}{$\exp$} & 0,000352 & 6,44E06 \\
\hline & $(0,000)$ & $(0,983)$ \\
\hline \multirow[t]{2}{*}{ imp } & 0,0883685 & 0,047975 \\
\hline & $(0,999)$ & $(0,451)$ \\
\hline \multirow[t]{2}{*}{ inf } & 52,23905 & 7,322916 \\
\hline & $(0,826)$ & $(0,943)$ \\
\hline \multirow[t]{2}{*}{$\mathrm{cg}$} & 30,65415 & 361,7637 \\
\hline & $(0,000)$ & $(0,711)$ \\
\hline \multirow[t]{2}{*}{ emp } & 28,94179 & 100,7142 \\
\hline & $(0,000)$ & $(0,018)$ \\
\hline \multirow[t]{2}{*}{ des } & $-10,6413$ & $-366,598$ \\
\hline & $(0,001)$ & $(0,017)$ \\
\hline \multicolumn{3}{|c|}{ P-valor en paréntesis } \\
\hline \multicolumn{3}{|c|}{$\begin{array}{l}{ }^{*} \text { Fixed effects }{ }^{*} \text { Between effects } \\
\text { Nivel de significancia de las estimaciones } 5 \%\end{array}$} \\
\hline
\end{tabular}

En cuanto a las variables sociales, la tasa de desempleo y de empleo resultaron ser significativas. El crecimiento de la población también es significativo y está relacionado positivamente con la inversión extranjera directa. La única variable social significativa es el crecimiento de la población.

Los signos de los coeficientes arrojados por los resultados de la estimación fueron los esperados. Al mismo tiempo, se evidencia que la inversión extranjera ha sido creciente en el periodo analizado, lo que se debe en gran parte a la relación que existe entre ésta y las variables independientes consignadas en las estimaciones econométricas realizadas.

\section{Conclusiones}

Los resultados muestran que el porcentaje del PIB destinado al gasto militar es significativo y tiene una relación positiva con la inversión extranjera directa, debido a que la IED determina su comportamiento en gran medida a la confianza y estabilidad que exista en un país.

26 Datos fuente. Datos tomados de DNP y Banco de la República, cálculos de los autores. 
La deuda externa tiene relación positiva y significativa con la IED, lo cual puede explicarse a partir de la capacidad de endeudamiento que un país posee y por lo tanto, lo atractivo que se forma para a los inversionistas. La tasa de alfabetismo, tiene relación positiva y significativa con la variable dependiente, lo que se explica por la mayor atracción que tiene hacia un inversionista un país cuyos niveles de educación pueden ser aprovechados, en mayor investigación de procesos y manejo de rentabilidades.

En el caso colombiano, las variables de comercio internacional, exportaciones, importaciones y balanza comercial, resultaron tener una relación significativa con respecto a la IED. Adicionalmente, se evidencia la existencia de una relación positiva entre la tasa de interés dtf y la formación bruta de capital. El comportamiento de la IED fue estable y tuvo incrementos en el periodo analizado, lo que se debe en gran parte a la relación que existe entre ésta y las variables independientes consignadas en las estimaciones econométricas realizadas. Los resultados obtenidos evidencian la relación positiva entre la tasa de interés dtf y la formación bruta de capital. Pero, lo cierto es que la tidtf es significativa sólo en la estimación de regresión robusta, mientras que la FBK es significativa en ambas estimaciones. El comportamiento de la IED en Colombia ha tenido un comportamiento estable con variaciones en el periodo 2000 - 2004 y el crecimiento de la economía ha sido creciente desde el 2001 - 2002. Dicho crecimiento, sobre todo en los últimos años, se soporta en los sectores de la construcción, manufactura y comercio.

Los resultados arrojados por las estimaciones econométricas muestran que las condiciones de la inversión extranjera directa se asocian en mayor medida a las características económicas que a las características propias de la situación de Conflicto Armado Interno. La cesación del conflicto generaría que se incentivara aún más la inversión extranjera directa e impulsaría el crecimiento económico en el país, lo que llevará a una mayor productividad y mejor calidad de vida para la sociedad colombiana.

En Colombia, las políticas gubernamentales han sido direccionadas hacia la disminución del conflicto tratando de acercarse cada vez más a la posibilidad de alcanzar su cesación definitiva. En el mismo sentido, las políticas del gobierno han buscado orientar la economía hacia niveles altos de crecimiento para lograr así una mayor estabilidad económica y confianza financiera en el país. Los resultados encontrados al realizar el documento reafirman la importancia de elaborar estudios de análisis que integren la economía con otras áreas sociales, de manera que se logre contextualizar la teoría económica con la realidad política y social, tanto a nivel nacional como a nivel internacional, buscando soluciones cada vez más eficaces que reflejen mejor calidad de vida de la sociedad mundial.

\section{Referencias}

Anuario de Asia Pacífico, 2006. "Conflictos Activos en Asia Pacífico en el 2006". [Disponible en Internet]. http://www. anuarioasiapacifico.es/anuario2006/pdf/021Conflictos.pdf

Apartado Estadístico del Banco Mundial, (2008). [Disponible en Internet]: http://datos.bancomundial.org/pais

Banco de la República. Series estadíticas. [Disponible en Internet]: http://www.banrep.gov.co/seriesestadisticas

Banco Mundial. http://www.bm.org

Baracaldo, D. et al., (2005). "Crecimiento economic y flijos de inversion extranjera directa. Bogotá, Universidad Externado de Colombia".

Bayer, R \& Rupert, M. (2004). "Effects of Civil Wars on International Trade, 1950-92". En: Journal of Peace Research, 41: 699-713.

Borrero, A., (2006). "Un conflicto armado en Colombia: un conflicto en crisis". En: Memorias Acnur

Calderon - Rossell, J., (1985). "Towards the theory of foreign direct investment". En: Oxford Economic Papers, 37:282-291.

Convenios de Ginebra del 12 de agosto de 1949. [Disponible en Internet]: http://www.hchr.org.co/documentoseinformes/ documentos/html/pactos/prot_II adicional_convenios_ginebra.html

Decreto 2080 de 2000. [Disponible en Internet]: http://www.mincomercio.gov.co/econtent/documentos/normatividad/decretos/decreto 2080 2000.pdf

Eriksson, M. et al. (2003). "Armed Conflict, 1989-2002". En: Journal of Peace Research, 40: 593-607.

Fondo Monetario Internacional. http://www.fmi.org

Gleditsch, N. et al. (2005). "Dataset Armed Conflict 1946-2005". En: Journal of Peace Research, 39(5): 615-637.

Gleditsch, N. et al. (2002). "Armed Conflict 1946-2001: A New Dataset". En: Journal of Peace Research, 39: 615-636.

Index of Economic Freedom, 2008. "Apartado de Haití". En: The Herital Foundation and The Wall Stret Journal. [Disponible en Internet]

Lacina, B. (2004). "From Side Show to Centre Stage: Civil Conflict after the Cold War". En: Security Dialogue, 35: 191-205.

Lalwani, M., (2002). "The impact of foreign direct investment on domestic savings and investment in selct developing economies". En: International Studies, 39: 79. 


\section{PRESENCIA DE CONFLICTO ARMADO INTERNO Y SU EFECTO EN LA INVERSIÓN EXTRANJERA DIRECTA: \\ TENDENCIA MUNDIAL Y PERSPECTIVAS PARA COLOMBIA (2001-2007)}

Lombard, F., (1978). "Screening foreign direct investment in LCDs: Empirical findings of the Colombian case (1967-1975)". En: Journal of international business studies, 9:66-80.

López M. et al. (2006). "Síntesis y perspectivas del contexto macroeconómico colombiano 2006-2007". Perfil de Coyuntura Económica, Edición diciembre 2006 pp. 5-48

Martin, S. (2005). "El auge de la Inversión Directa Extranjera (IDE) en Asia". En: Carta de Asia-Economía, 145.

Mansoob, S., (2002). "Conflict, civil war and underdevelopment: an introduction". En: The Journal of Peace Research, 39 387-393.

Murdoch, J. \& Sandler, T. (2002). "Economic Growth, Civil Wars, and Spatial Spillovers". En: Journal of Conflict Resolution, 46: $91-110$.

Nigh, D. \& Schollhammer, H., (1987). "Foreign direct investment, political conflict and co-operation: the asymmetric response hypothesis". En: Managerial and decision economics, 8:307-312.

Organización de las Naciones Unidas, ONU. [Disponible en Internet]: http://www.onu.org

Pizarro L. E. (2004). "Una Democracia Asediada. Balance y perspectivas del conflicto armado en Colombia". Grupo Editorial Norma, Bogotá, Colombia.

Pickering, J. \& Kisangani, E. (2006). "Political, Economic, and Social Consequences of Foreign Military Intervention". En: Political Research Queatrly, 59: 363-376.

Pindyck S. Robert y Rubinfeld R. Daniel, (1998). Econometría. Modelos y Pronósticos. Capitulo 9: Estimación de una sola ecuación. Cuarta edición.
Proexport. Estadísticas. [Disponible en Internet]: http://www. proexport.com.co/VBeContent/home.asp?language=SP\&id company $=16$

Ramirez, C. \& Florez, L., (2006). "Apuntes de inversión extranjera directa: definiciones, tipología y casos de aplicación coIombianos". En: Apuntes de Economía, Universidad ICESI, 8:2-26.

Restrepo, J. (2001). "Análisis económico de conflictos internos". En: Ideas para la Paz. 1-35.

Reuber, G., et al. (1973). "Private Foreign Investment in Development". En: Clarendon Press: Oxford

Reynal-Querol, Marta. (2002). "Ethnicity, Political Systems, and Civil Wars". En: Journal of Conflict Resolution, 46: 29-54.

Sambanis, N. (2001). "Do Ethnic and Nonethnic Civil Wars Have the Same Causes?: A Theoretical and Empirical Inquiry (Part 1)". En: Journal of Conflict Resolution, 45: 259-282.

UNCTAD, (Conferencia de las Naciones Unidas sobre comercio y desarrollo). "La inversión extranjera directa en África". En: Publicaciónes.[Disponible en Internet] http://www.unctad. org/templates/Page.asp?intltemID =3524\&lang =3

UNCTAD, (2005). "Economic Development in Africa: Rethinking the role of foreign investment". En: Publicaciones, unctad. [Disponible en Internet]

http://www.unctad.org/Templates/webflyer.asp?docid=6056\&in tltemID =1397\&lang =3

Valencia, G. (2006). "La economía frente al conflicto armado interno colombiano, 1990-2006". En: Perfil de Coyuntura Económica, 8: 141-174.

Wallensteen, P. \& Sollenberg, M. (2001). "Armed Conflict, 19892000". En: En: Journal of Peace Research, 38: 629-644 


\section{Anexo I}

Cuadro No. 1. Fuente de las Variables empleadas

\begin{tabular}{|c|c|}
\hline VARIABLE & FUENTE \\
\hline Inversión Extranjera Directa (mundial) & Apartado estadístico del Banco Mundial. \\
\hline Inversión Extranjera Directa (Colombia) & Departamento Nacional de Planeación. Proexport. \\
\hline Crecimiento del Producto Interno Bruto (mundial) & Apartado estadístico del Banco Mundial. \\
\hline Producto Interno Bruto (Colombia) & Banco de la República \\
\hline Inflación (mundial) & Fondo Monetario Internacional \\
\hline IPC (mundial) & Fondo Monetario Internacional \\
\hline Exportaciones como porcentaje del PIB (mundial) & Apartado estadístico del Banco Mundial. \\
\hline Importaciones como porcentaje del PIB (mundial) & Apartado estadístico del Banco Mundial. \\
\hline Exportaciones (Colombia) & Departamento Nacional de Planeación. \\
\hline Importaciones (Colombia) & Departamento Nacional de Planeación. \\
\hline Balanza Comercial & Dane \\
\hline Deuda externa (mundial) & Apartado estadístico del Banco Mundial. \\
\hline Tasa de Interés Activa (Colombia) & Banco de la República \\
\hline Tasa de Interés de Colocación (Colombia) & Banco de la República \\
\hline Tasa de Interés DTF (Colombia) & Banco de la República \\
\hline Formación Bruta de Capital & Banco de la República \\
\hline Presencia de Conflicto Interno & $\begin{array}{l}\text { Geditsch et al. (2005), Ericksson et al. (2003), Geditsch et al. } \\
\text { (2002) y Wallensteen y Sollenberg (2001) publicados en el Jour- } \\
\text { nal of Peace Research. }\end{array}$ \\
\hline Intensidad del conflicto & Wallensteen (2001) \\
\hline Porcentaje del ingreso utilizado para el gastomilitar & Apartado estadístico del Banco Mundial \\
\hline Población & Dane \\
\hline Coeficiente de Gini & Departamento Nacional de Planeación. \\
\hline Tasa de crecimiento de la población (mundial) & Apartado estadístico del Banco Mundial \\
\hline Tasa de Alfabetismo (mundial) & $\begin{array}{l}\text { Apartado estadístico del Banco Mundial y Fondo Monetario In- } \\
\text { ternacional }\end{array}$ \\
\hline Tasa de Desempleo (Colombia) & Departamento Nacional de Planeación. \\
\hline Tasa de Empleo (Colombia) & Departamento Nacional de Planeación. \\
\hline
\end{tabular}




\section{Anexo II \\ Conflictos armados internos en el mundo}

Un conflicto armado interno se presenta entre el gobierno de un Estado y grupos de oposición interna sin la intervención de otros Estados. De igual manera, los conflictos armados pueden ser clasificados según su intensidad, teniendo en cuenta un rango del número de homicidios que se presentan en dichos enfrentamientos, a saber: conflicto armado menor ${ }^{23}$, conflicto armado intermedio ${ }^{24}$ y gue$\mathrm{rra}^{25}$. Dicha clasificación es tomada en cuenta en este documento con el fin de construir una variable de conflicto que complemente la estimación econométrica, Wallensteen, P. \& Sollenberg, M. (2001).

\section{Clasificación por naturaleza y ubicación}

En el periodo 2001-2007, según las bases de datos y literatura revisada, se encontró la presencia de 38 conflictos armados internos en el mundo, ubicados en 3 continentes: Asia, África y América. Sin embargo, la concentración de los Conflictos Armados Internos es más evidente en África y en Asia con 36 de los 38 conflictos existentes en ese periodo.

África, por su parte, se ha caracterizado por los legados coloniales y traumatismos en su estructura social y política por las transiciones y divisiones abruptas de territorio realizadas por los países colonizadores ${ }^{26}$. Las divisiones y transiciones bruscas han logrado crear inestabilidad política, económica y social en África, como bien se puede ver en Ruanda, Somalia y Burundi, donde la situación de con- flicto ha sido consecuencia de las ansias de descolonizar y el efímero criterio de afrontar la separación del país colonizador (Manssob, 2002).

Por otro lado, Asia tiene aspectos diferenciadores en cuanto a la variedad de causas de sus conflictos. A pesar de ello, el desarrollo de las naciones ha sido determinado en gran parte por creencias y legados ancestrales que trazaron rivalidades a lo largo de la historia.

Las diferentes culturas, etnias y creencias han sido factor fundamental de la gran mayoría de conflictos en el continente asiático. Ahora bien, los Conflictos Armados Internos se han tornado cada vez más complejos por los diversos matices sociales, económicos y políticos que influyen en los mismos. De todos modos, en la mayoría de ellos es clara la naturaleza de su inicio, esto es, el por qué surgió en un comienzo la confrontación. Así, la naturaleza de los conflictos se clasificó en causas étnicas y/o religiosas, políticas/ideológicas y por recursos o territorio ${ }^{27}$.

De esta manera, se determinó que de los 38 Conflictos Armados Internos encontrados, 11 son de características étnicas y/o religiosas, 17 de carácter político/ideológico y 10 existen a raíz de una lucha por recursos y/o territorio. Adicionalmente, es importante mencionar que 4 países de Asia (China, India, Pakistán e Indonesia) poseen dos conflictos

23 Por lo menos 25 muertes por enfrentamientos o en relación a ellos, por año y menos de 1,000 muertes por enfrentamientos durante todo el conflicto.

24 Por lo menos 25 muertes por enfrentamientos o en relación a ellos, por año y un acumulado total de por lo menos 1,000 muertes, pero menos de 1,000 por año.

25 Por lo menos 1,000 muertes por enfrentamientos o en relación a ellos, por año.

26 Las naciones afectadas por la presencia de conflictos tienen estructuras sociales débiles, las cuales, en muchos casos, son consecuencia de la herencia colonial, (Manssob, 2002).

27 Clasificación de los Conflictos Armados Internos en el Mundo: (i) Étnicos/Religiosos: China, Pakistán, India, Sri Lanka, Indonesia, Tailandia, Burundi, Sudan, Ruanda, Nigeria, Costa de Marfil; (ii) Políticos/Ideológicos: Afganistán, Myanmar Bangladesh, Nepal, Irán, Iraq, Laos, Uzbekistán, Timor Oriental, Angola, R.D. Congo, Chad, Somalia, R. Centroafricana, Uganda, Colombia y Haití; (iii) China, Israel, India, Indonesia, Pakistán, Rusia, Turquía, Filipinas, Senegal y Etiopía.(Clasificación realizada por el autor a partir de Wallensteen (2001) Ericksson et al. (2003), Geditsch et al. (2005). 
al interior de sus fronteras, uno de ellos de carácter étnico y/o religioso y el otro por lucha de recursos y/o territorio.

Se considera que un conflicto de características étnicas, religiosas o de minorías es aquel que surge a partir de inconformismo, violencia, tortura, rechazo y/o abuso de minorías étnicas y religiosas que habitan dentro de un Estado ocasionadas por parte de grupos de personas que no toleran su presencia dentro del espacio territorial por motivos de etnia, raza, nacionalidad o religión ${ }^{28}$.

Igualmente, un conflicto de carácter político/ideológico se define como la lucha por el poder político o la implementación total de una ideología determinada, los cuales son factores que causan la mayoría de los conflictos en el mundo. En dichos conflictos se originan grupos que pretenden derrocar el gobierno establecido en el momento y por lo general, utilizan a la población para que se unan y así armar milicias que combatan los ejércitos institucionales (Anuario Asia-Pacífico, 2006).
Por otro lado, un conflicto por acceso a recursos es aquel que se da cuando dos o más actores entran en conflicto para compensar un desigual acceso a los recursos. Este tipo de conflicto que tiene como características la competencia entre actores para conseguir el Gobierno legítimo de un territorio determinado, por el cual luchan y además, en la mayoría de los casos tiene gran variedad y tamaño en recursos naturales ${ }^{29}$.

El conflicto colombiano tiene características diversas, es decir, la naturaleza es principalmente política e ideológica debido a que de las ideas políticas de los insurgentes nació lo que son hoy los grupos armados ilegales. Ahora bien, el componente económico que ha caracterizado el conflicto desde la década de los 80 juega un papel fundamental y se torna en multifacético con diferentes actores involucrados desde las guerrillas, paramilitares, carteles y hasta campesinos que viven del negocio de la droga.

Según lo definido en el Anuario de Asia Pacífico, Conflictos Activos en Asia Pacífico en el 2006.

33 Anuario de Asia Pacífico, Conflictos Activos en Asia Pacífico en el 2006. 